\title{
The development of a waste heat recycle power generation system
}

\author{
David King Jair ${ }^{\mathrm{a}}$, Ming Chun Hsieh ${ }^{\mathrm{b}}$,

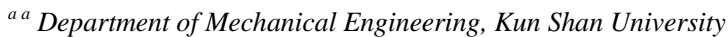 \\ ${ }^{b b}$ Department of Electronic Engineering, Kun Shan University
}

\begin{abstract}
In this study, a novel waste heat recycle power generation system is developed by using an integrated design to give higher power generation efficiency. The system consists of thermoelectric generator (TEGs), heat collecting sheets and absorbing layer. After the construction of the generator, the performance of the generator was experimentally evaluated. A collaborative enhancement of output power for TEG modules are obtained and the overall output power has been improved by the optimized design of the system. Based on the experiments, after heating 20 minutes, the temperature difference of TEG can reach $53^{\circ} \mathrm{C}$, and the output voltage and current are $35 \mathrm{~V}, 0.56 \mathrm{~A}$ respectively. The total amount of generated power is enhanced to $20 \mathrm{~W}$. Furthermore, the prototyped of the waste heat recycle power generation system is relatively small and cheap. After the fabrication and testing of the prototype, this system has been proved feasible for practical application.
\end{abstract}

Keywords: Renewable energy, thermoelectric generator, waste heat, heat collector

\section{Introduction}

Sources of renewable energy are a major global concern for researchers. The rising cost of fossil fuel and their associated global environmental impact has led researchers to develop methods of replacing the use of fossil fuels with alternative renewable and sustainable energy sources [1-4]. Efficient sources of renewable energy (e.g., solar, wind, wave, tide, acoustic, geothermal, heat, and kinetic energy) are therefore being explored and being developed as alternative solutions to many current and future energy problems. The most interesting of these energies are wind power and solar energy. However, the use of solar energy technologies has a very high cost of the shortcomings. The limited life of solar panels is about 10-20 years and the production required the use of a large number of silicon, germanium, and boron may cause pollution in other areas. On the other hand, the wind power energy has high installation cost, big noise, locations and wind farm requirements. For businesses, reducing costs while improving environmental performance is an increasing priority. With the high cost and environmental impact of fossil fuels, heat energy is a precious commodity that cannot be wasted.

Any exhaust gas stream with temperatures above $300^{\circ} \mathrm{C}$ has the potential for significant waste heat recovery. Consumers of waste heat energy can be found in almost any facility and are easy to locate. Typical examples include plant process heating, combustion air pre-heating, boiler feedwater pre-heating, and building heat. In addition to savings in everyday fuel consumption, many facilities can market and sell carbon credits back to industry. State and / or federal funding is often available for waste heat recovery projects helping to reduce capital costs and expedite system payback. Generally, large factories use heavy oil and coal as fuel. The process will inevitably produce a lot of waste heat. This waste heat, if allowed to discharge, will become the overall energy loss. If the waste heat recovery unit is used to recover the waste heat from the industrial facilities, it can be used as the fuel for the whole plant heat and

\footnotetext{
* Manuscript received October 24, 2017; revised March 14, 2019.

Corresponding author. E-mail address: styxking@gmail.com.

doi: 10.12720/sgce.8.3.325-331
} 
the Cogeneration system to improve the overall resource and energy efficiency. Taiwan started with manufacturing, where many traditional industries consume large amounts of energy and generate some significant waste heat in the manufacturing process, yet the rate of waste heat recovery is low, resulting in significant energy waste and carbon dioxide emissions.

Recently, waste heat harvesting by using thermoelectric generator (TEG) in various cases have been reported by research groups worldwide, such as in applications of pipelines, furnaces [5], geothermal, solar heat [6], [7], and automobile engines [8]-[11]. It becomes more and more important to recycle waste energy into useful power, and overcoming the material cost constitutes a problem to be solved through technological progress. TEGs are devices which convert wasted excess heat energy and convert it into a reusable form of energy. The thermoelectric effect is the direct conversion of temperature differences to electric voltage and vice versa. A thermoelectric device creates voltage when there is a different temperature on each side. Also, the TEG is scalability, it can be applied to any size heat source from a water heater to a manufacturers equipment, and low production cost; therefore it is very suitable to be used for recycling waste heat from the industries under $300^{\circ} \mathrm{C}$ for power generation. However, the primary challenge of the thermoelectric power generation is its relatively low heat-to-electricity conversion efficiency. In this paper, a system to recover waste heat has been designed, simulated and fabricated to achieve the objective of recovered energy, as explained in the following sections.

\section{Design and Fabrication}

The TEG is a semiconductor device which converts heat energy directly into electrical energy. A TEG consists of number of cubical semiconductor blocks and it appears like a plate. The heat is applied to one side of it and the other side is kept relatively cool. The principle of TEG states that the difference in temperature in two sides of the TEG generates electricity, as shown in fig.1. Hence, according to Thomas J. Seebeck in 1821, a potential difference could be produced by a circuit made from two dissimilar wires when one of the junctions was heated. This is called Seebeck effect [12]. The eletromotive force is proportional to the temperature difference. The potential difference is given as: $\mathrm{V}=\alpha \Delta \mathrm{T}$. One thermoelectric module comprise of two different semiconductor materials also known as Seebeck cells or thermo elements each of p-type and n-type cells which are connected electrically in series and thermally in parallel. In 1834, J. Peltier observed the reverse Seebeck effect known as Peltier effect. According to Peltier effect the passage of an electric current through a thermocouple produces a small heating or cooling effect depending on its direction. The interdependency between Seebeck effect and Peltier effect was later determined by $\mathrm{W}$. Thomson known as Thomson effect consists of reversible heating or cooling when there is presence of both a flow of electric current and a temperature gradient [13].

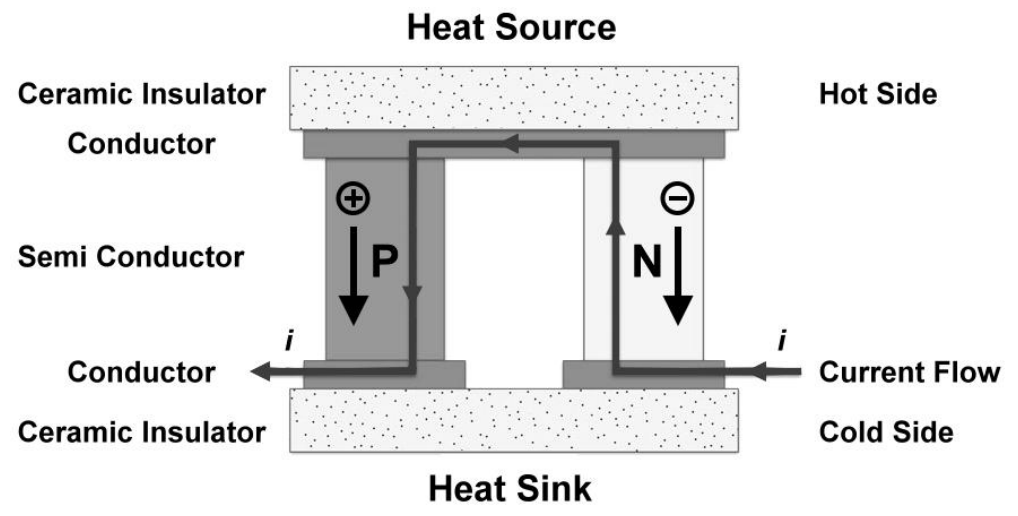

Fig. 1. The schematic of a thermoelectric generator.

The waste heat recovery system is designed for exhaust chimney of stove. TEG modules (Marlow, TG12-801L) are connected electrically in series but thermally in parallel, and these modules are arranged 
on the four surfaces of aluminum heat exchanger through which the exhaust gas is passed. The main component of the system are the TEGs and heat sinks. A plurality of groups of TEGs are arranged around a set of demountable heat flow channels, and the heat is led to the hot side of the thermoelectric generator (TEG) by means of a thermally conductive material and at the other side (cold side) of the thermoelectric generator. The heat sinks were designed to transfers the heat to keep the cold side at a low temperature.

Figure 2 shows the schematic of the waste heat recovery system. The TEG is tightly attached to the detachable heat flow passage through the conductive material. When the heat flow through the tunnel, it produces a temperature difference between both ends of the TEGs, then generate electricity. The conversion efficiency of TEG is related to the temperature difference between hot and cold sides. That is, whether it can keep the cold side at room temperature. Therefore, the effectiveness of the heat dissipation device is critical. Furthermore the power output of a TEG module without any heat collector is lower, to obtain high quality power output, it is feasible to insert a plate-fin heat sink into a chimney tunnel, which can increase the convective heat transfer area and reduce thermal resistance from the flue gas to the TEG module.
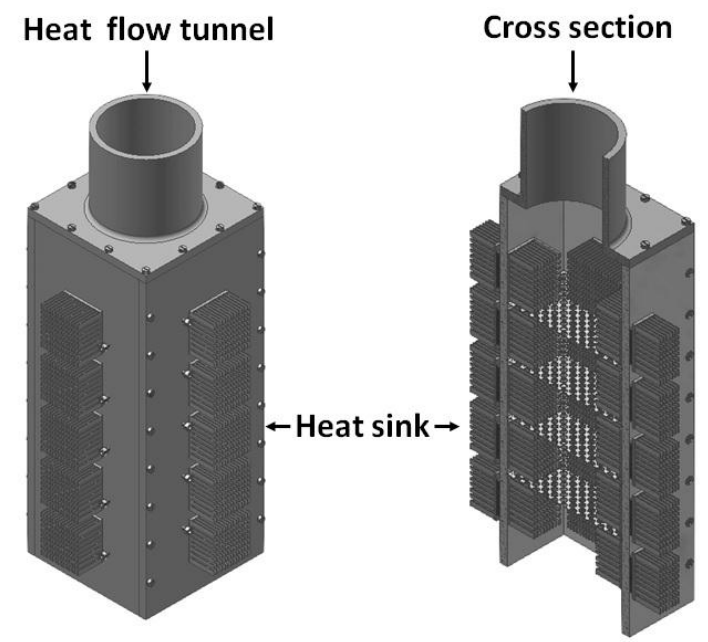

Fig. 2. The schematic of the waste heat recovery system.

The schematic of the thermoelectric module with a plate-fin heat sink for waste heat recovery system is shown in fig. 3 .
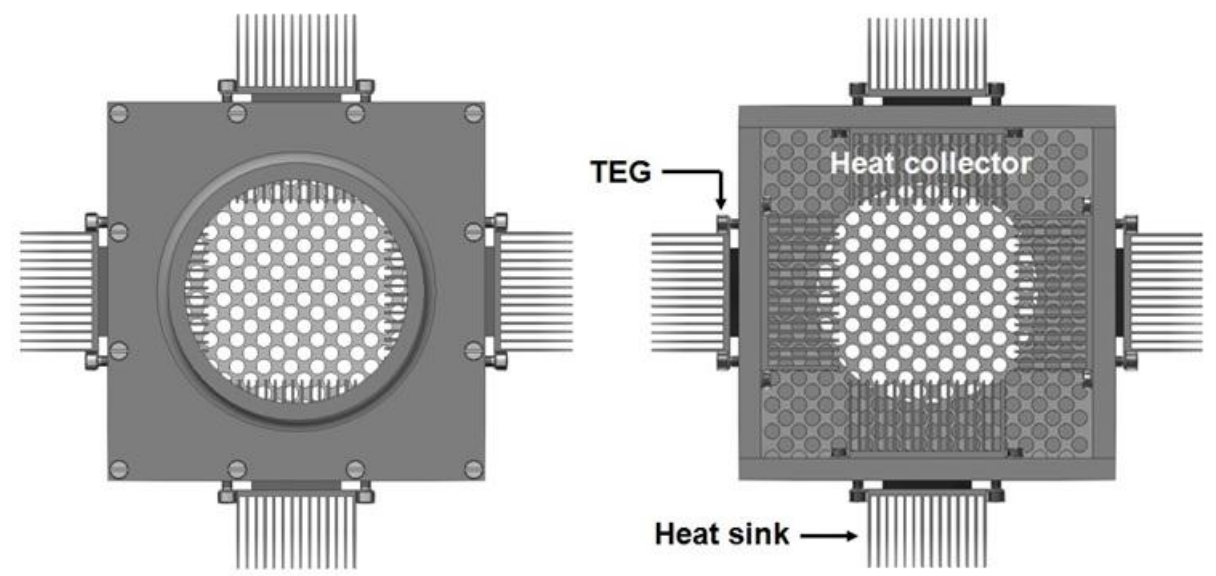

Fig. 3. Schematic of the thermoelectric module with a plate-fin heat sink for waste heat recovery system, top (left) and bottom (right) view. 
Some measuring instruments are equipped to construct this experimental setup. T-type thermal couples are embedded in hot and cold sides of TEG modules to record temperature while measuring. The ammeter and the voltmeter are connected to measure the current in the circuit and voltage cross the external load resistor respectively.
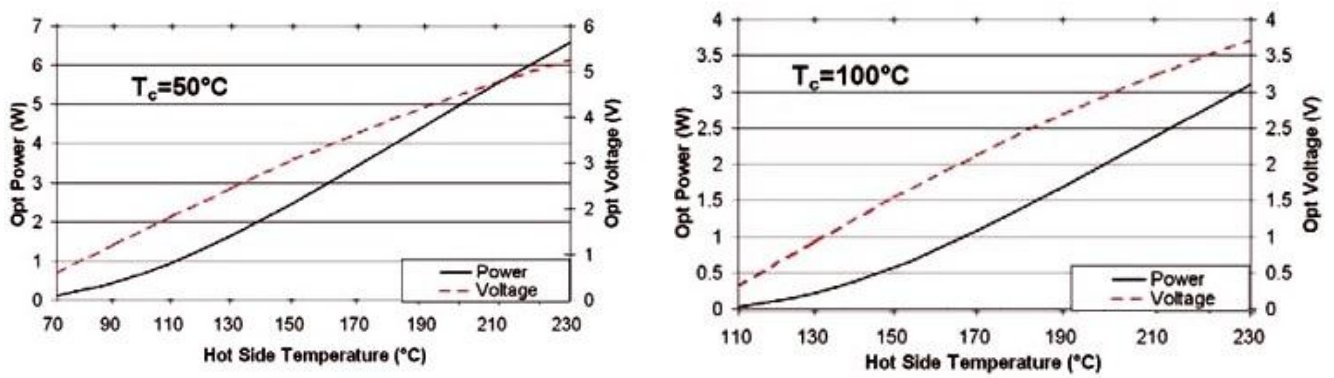

Fig. 4. The characteristic curve between the voltage and power efficiency for a single thermoelectric material [14]

We use a thermoelectric chip for TG12-801L, its size is $40 * 45 * 3.5 \mathrm{~mm}$. Fig. 4 shows the characteristic curve between the voltage and power efficiency for a single thermoelectric material. As shown in fig.4, it can be seen that when the cold side temperature is maintained at $50{ }^{\circ} \mathrm{C}$ and the hot side temperature is $230{ }^{\circ} \mathrm{C}$, the output voltage can reach $5 \mathrm{~V}$ and the output power is close to $6 \mathrm{~W}$. The cold side temperature is maintained at $100{ }^{\circ} \mathrm{C}$, the hot side temperature is $230{ }^{\circ} \mathrm{C}$, the output voltage can reach $3 \mathrm{~V}$, the output power efficiency is close to $4 \mathrm{~W}$. The greater the temperature difference at both sides of the TEG the better the power generation effect, and the higher the output power; so in the cooling part is very important.

\section{Research Method}

To estimate the output performance of this case study, the waste heat recovery system is setup, as shown in Fig. 5. The copper heat sinks upon the TEG module should decrease the temperature at the cold side of the TEG to generate a larger temperature difference. 20 19-fin structured aluminum heat sinks are assembled oppositely as a heat exchanger for waste gases passing through. The fin structures are made up heat exchanging more effectively than that without fin structures which is discussed in previous study. There are 20 TEG modules sandwiched between heat sinks and the heat exchanger in this system. In order to reduce thermal contact resistance, a thermal interface material, thermal grease, was smeared uniformly on the both side of TEG module.
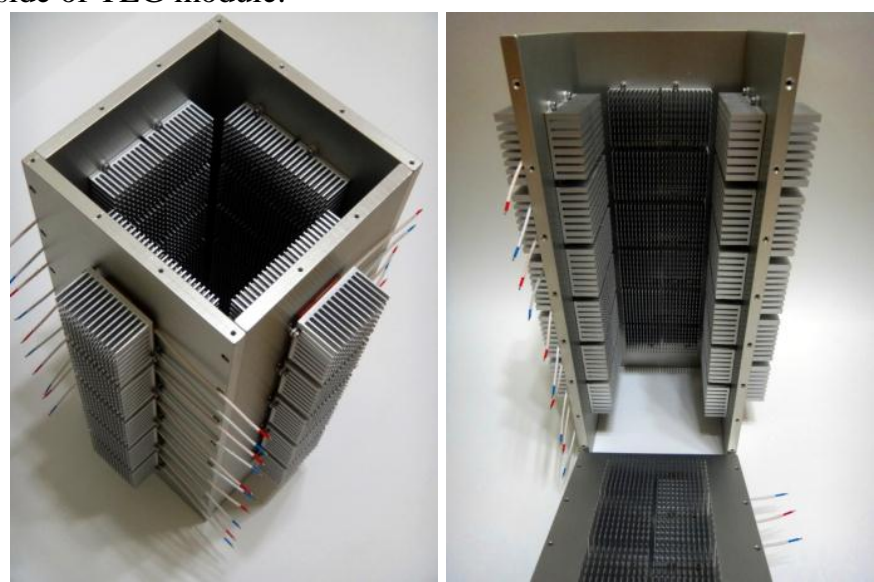

Fig. 5. The prototype of the waste heat recovery system 


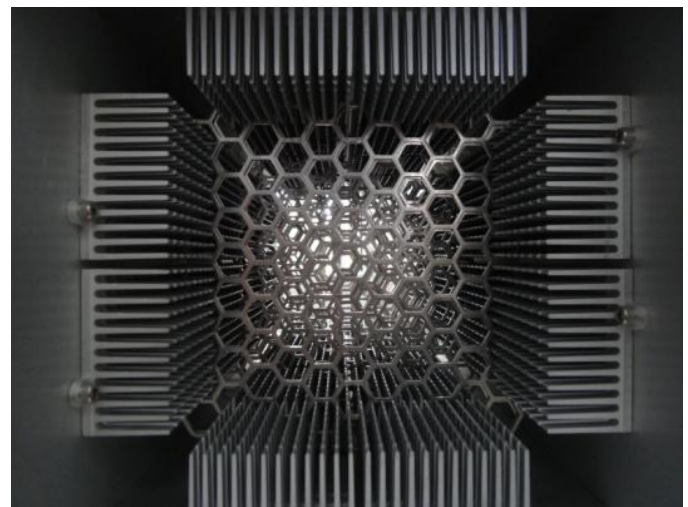

Fig. 6. The perforated metal sheets in the channel as thermal conduction layer

In order to avoid heat flow directly from the thermal convection to affect the absorption of thermoelectric chips we added perforated metal sheets as thermal conduction layer. After experimenting different diameter of holes, the $3 \mathrm{~mm}$ diameter perforated metal is the most suitable as shown in fig.6. Putting four thermal conduction layers together and making it a tunnel for heat flow, then install a thermal module inside the tunnel and connect it to the vent. After that, put a custom heat-absorbing plate in it, which will increase the amount of exhaust gas absorbency, containing the temperature difference on the hot side of the cooling chip. In addition, analytical results will be validated through a series of experiments to demonstrate the usefulness of the system. Power outputs with the system are also determined for different heating time.

\section{Experimental Results}

The electrical testing has been carried out. In order to study the thermal distribution of the actual heat flow in the heat pipe, we divided the device into three parts; which are the upper, the middle and the lower part. Figure 7 shows the temperature difference at different position of TEG as a function of heating time without thermal conduction layer. After heating 15 minutes, the maximum temperature difference of the the upper TEG is $27^{\circ} \mathrm{C}$, the maximum temperature difference in the middle and lower TEG are $21^{\circ} \mathrm{C}$, and at the lower TEG temperature difference is $14^{\circ} \mathrm{C}$.

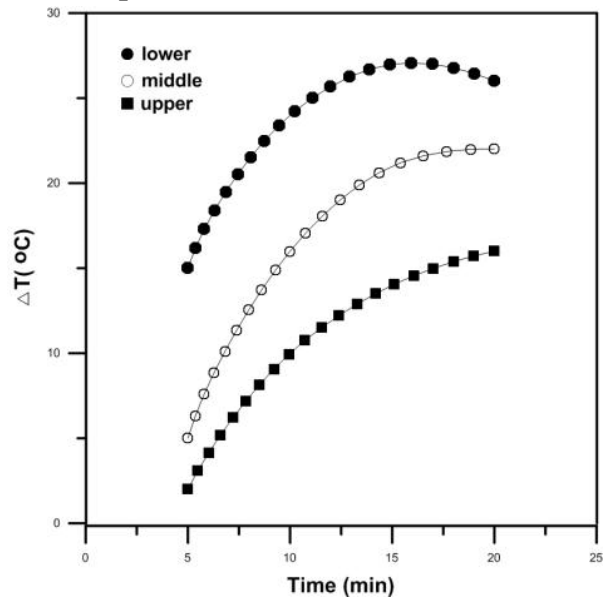

Fig. 7. The temperature difference at different position of TEG as a function of heating time. 


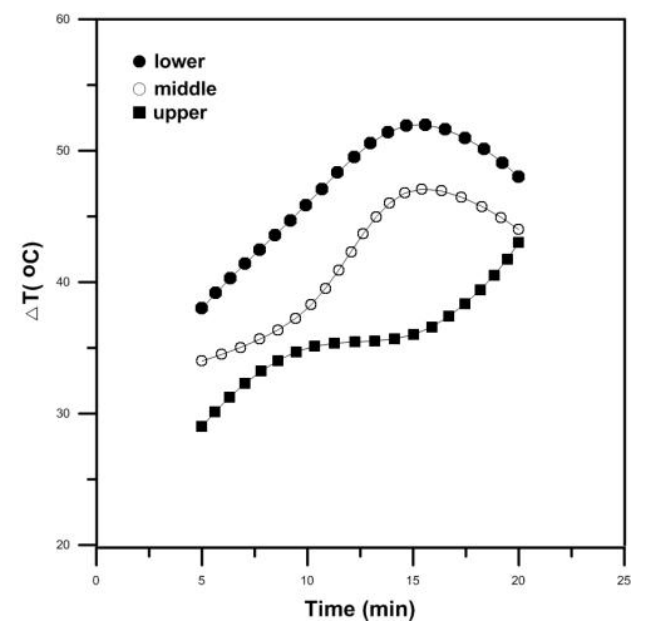

Fig. 8. The temperature difference at different position of TEG as a function of heating time.

Figure 8 shows the temperature difference at different position of TEG as a function of heating time with thermal conduction layer. After heating 15 minutes, the maximum temperature difference of the the upper TEG is $53^{\circ} \mathrm{C}$, the maximum temperature difference in the middle and lower TEG are $47^{\circ} \mathrm{C}$, and at the lower TEG temperature difference is $36^{\circ} \mathrm{C}$. It can be seen that increasing the heat collecting layer can effectively conduct the heat flow to the TEG. As heating time increases, the temperature difference between the two sides of the TEG begins to decrease because of the conduction of heat flow from the hot side to the cold side. Therefore, the thermal dissipation of the cold side is very important

Figure 9 illustrate the output voltage and current as a function of heating time. In fig.9, the black dots represent the relationship between the voltage and the heating time, while the white dots represent the diagram of the current over time. As the graph shows, the voltage and current increase with heating time, and after 20 minutes, the voltage is increasing to $35 \mathrm{~V}$, while the current is $0.65 \mathrm{~A}$. The overall power generation reaches $20 \mathrm{~W}$.

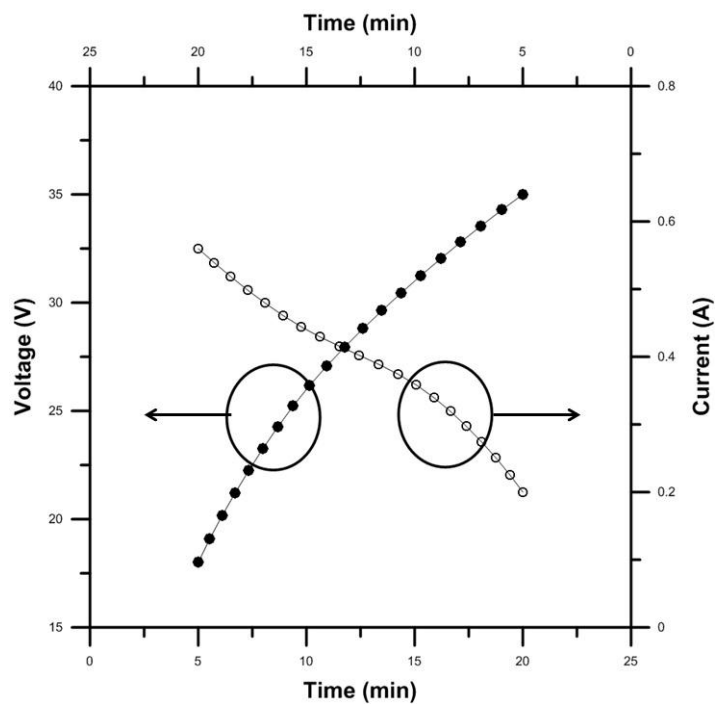

Fig. 9. The relationship of performance of TEG with heat time

From the experimental results, the design of heat collectors makes thermal distribution more uniform than that without the heat collectors. Thus the performance of TEG modules on both sides is 
improved. When the absorbing layer is loaded, the thermal energy can be absorbed by the absorbing layer and translated into heat at high efficiency. And heat absorption is more effective to produce higher temperature difference across the TEG. In this experimental condition, it is very difficult to maintain a fixed cold-side temperature (Tc). That is, with the increasing waste heat energy from the heating rate, the values of temperature difference, hotside's temperature and coldside's temperature all get increased in this specific case study.

\section{Summary}

In this paper, a novel waste heat recycle power generation system is developed by using an integrated design to give higher power generation efficiency. The system consists of TEGs, heat collecting sheets and absorbing layer. The absorbing layer is used to collect thermal energy. Much more heat is collected to the hot side of TEG due to the design of heat collector. After the construction of the generator, the performance of the generator was experimentally evaluated. A collaborative enhancement of output power for TEG modules are obtained and the overall output power has been improved by the optimized design of the system.

The test is operating in electrical machines laboratory and essentially test to determine the characteristics of prototype generator. Based on the experiments, after heating 20 minutes the temperature difference of TEG i can reach $53^{\circ} \mathrm{C}$, the output voltage and current are $35 \mathrm{~V}, 0.56 \mathrm{~A}$, respectively. The output power approaching from the original $8 \mathrm{~W}$ raised to $20 \mathrm{~W}$. And the design of the increased heat conduction layer can be used to guide the heat flux to the thermoelectric chip and improve the efficiency of heat transfer. The higher the temperature of thermoelectric materials at both ends, the better the power generation and the higher the output power, so the part of the heat dissipation is very important. In the future, this research will improve the heat dissipation part in order to increase its power generation efficiency.

\section{References}

[1] Xi H, Luo L, Fraisse G. Development and applications of solar-based thermoelectric technologies. Renew Sustain Energy Rev. 2007; 11: 923-936.

[2] Omer AM. Focus on low carbon technologies: the positive solution. Renew Sustain. Energy Rev 2008;12:2331-57.

[3] Afshar O, Saidur R, Hasanuzzaman M, Jameel M. A review of thermodynamics and heat transfer in solar refrigeration system. Renew Sustain Energy Rev. 2012;16:5639-48.

[4] Thirugnanasambandam M, Iniyan S, Goic R. A review of solar thermal technologies. Renew Sustain Energy Rev, 2010;14:312-22.

[5] T. Ota, K. Fujita, S. Tokura, and K. Uematsu, "Development of thermoelectric power generation system for industrial furnaces," in Proc. Int. Conf.Thermoelectrics, 2006, pp. 354-357.

[6] Eisenhut C, Bitschi A. Thermoelectric conversion system based on geothermal and solar heat. in Proc. Int. Conf. Thermoelectrics, 2006: 510-515.

[7] Hasebe M, Kamikawa Y, and Meiarashi S. Thermoelectric generators using solar thermal energy in heated road pavement. in Proc. Int. Conf. Thermoelectrics, 2006, pp. 697-700.

[8] Haidar JG, and Ghogel JI. Waste heat recovery from the exhaust of low-power diesel engine using thermal electric generators. in Proc. Int. Conf. Thermoelectrics, 2001; 413-418.

[9] Yang J. Potential applications of thermoelectric waste heat recovery in the automotive industry. in Proc. Int. Conf. Thermoelectrics, 2005, pp. 155-159.

[10] LaGrandeur J. et al., Automotive waste heat conversion to electric power using skutterudite, TAGS, PbTe and BiTe. in Proc. Int. Conf. Thermoelectrics, 2006; 343-348.

[11] Talom HL and Beyene A. Heat recovery from automotive engine. Appl. Therm. Eng., 2009; 29(2-3): $439-444$.

[12] Goldsmid HJ. Applications of Thermoelectricity, Methuen Monograph, London, 1960.

[13] Mala R, Prasadb R, Vijayc VK, Vermad AR. The design, development and performance evaluation of thermoelectric generator (TEG) integrated forced draft biomass cookstove. Procedia Computer Science., 2015; 52: 723-729.

[14] II-VI Marlow, Inc. (2017). [Online]. Available: https://www.marlow.com/ 